\title{
Agua subterránea: tendencias y desarrollo científico
}

\author{
Lina M. Cerón, Jhon D. Sarria, Johan S. Torres y Jonathan Soto-Paz \\ Facultad de Ciencias Naturales e Ingeniería, Grupo de Investigación TERRANARE, Fundación Universitaria de San Gil - \\ UNISANGIL, Calle 7 No. 20 - 63, Yopal-Colombia. (correo-e: Iceron@unisangil.edu.co; jsarria@unisangil.edu.co; \\ jtorres@unisangil.edu.co; jsoto@unisangil.edu.co)
}

Recibido Jun. 23, 2020; Aceptado Ago. 21, 2020; Versión final Oct. 21, 2020, Publicado Feb. 2021

\begin{abstract}
Resumen
Este artículo analiza tendencias de investigación sobre aspectos técnicos en gestión del agua subterránea y presenta una postura crítica al respecto. El agua subterránea constituye una fuente de abastecimiento prometedora para diferentes usos acorde con su calidad. Esta temática ha sido considerada desde diferentes perspectivas técnicas que proporcionan un marco conceptual. Se emplearon herramientas bibliométricas como RefViz(®) y T-Lab(®) para procesar información recopilada de bases de datos especializadas teniendo en cuenta una ventana de observación de 10 años. Se encontró que la calidad del agua subterránea es un aspecto prioritario en investigaciones, así como la formulación de índices de calidad que sean aplicables a determinado contexto. Adicionalmente, los estudios hidrogeológicos que contemplen la variabilidad espacial y temporal ofrecen otra oportunidad de investigación que permite un mejor manejo del recurso. Se concluye que la integración de estas investigaciones puede contribuir a incrementar la eficiencia del uso del agua subterránea.

Palabras clave: índices de calidad; hidrogeología; acuífero; contaminación del agua; tendencias
\end{abstract}

\section{Groundwater: trends and scientific development}

\begin{abstract}
This present study analyzes research trends on technical aspects of groundwater management while presenting a critical view. Groundwater is a promising supply source for different uses according to its quality. This topic has been considered from different technical perspectives that provide a conceptual framework. Bibliometric tools such as RefViz $(\Theta)$ and T-Lab $(\circledR)$ are used to process groundwater information that is collected over a 10-year observation window from specialized databases. The results show that both groundwater quality and the development of quality indices that are applicable to a given context are priority research areas. In addition, hydrogeological studies that contemplate spatial and temporal variability offer research opportunities for better groundwater management. It is concluded that integration of these research approaches can contribute to increasing the efficiency of groundwater use.
\end{abstract}

Keywords: quality indices; hydrogeology; aquifer; water pollution; trends 


\section{INTRODUCCIÓN}

El acceso al agua es un derecho humano fundamental y representa un factor esencial en el desarrollo integral y sostenible de la sociedad y su eficiente gestión es reconocida en los objetivos de desarrollo sostenible. El agua subterránea representa el $98 \%$ del agua dulce no congelada disponible como fuente de abastecimiento para múltiples usos dependiendo de sus características fisicoquímicas y biológicas (Subba Rao y Chaudhary, 2019). Este recurso es cuantitativamente mayor que el agua superficial, participa de numerosos procesos naturales y brinda innumerables servicios ecosistémicos.

Por sus características hidrogeológicas el agua subterránea presenta menor susceptibilidad a contaminación antrópica y a las variaciones ambientales, sin embargo, problemáticas como la creciente demanda del recurso y el aumento en el grado de contaminación se han convertido en asuntos de interés mundial (Kumar et al., 2015), considerando que una vez que un acuífero se ha contaminado, su "limpieza" se vuelve impracticable y los procesos de remediación implica altos costos económicos y sociales (Kalhor et al., 2019). En torno al agua subterránea, las investigaciones se han orientado en evaluar la calidad a través del estudio de parámetros fisicoquímicos y biológicos, como también evaluar las variaciones hidrológicas en términos espaciales y temporales que inciden en la gestión integral del recurso hídrico.

Este artículo presenta las tendencias de investigación en torno a los aspectos técnicos de la gestión del recurso hídrico durante el periodo 2010-2020. Para tal efecto, se emplearon herramientas bibliométricas que son fundamentales para la captación de información, su análisis y toma de decisiones. A partir del análisis de la información, se desarrolla una reflexión crítica sobre las tendencias de investigación aplicables al contexto de países en desarrollo que permita identificar brechas o vacíos de investigación en este tema.

\section{MATERIALES Y MÉTODOS}

La metodología usada para la vigilancia tecnológica se realizó acorde con Gaviria et al. (2019). Se realizó una búsqueda sistemática de información en una ventana de 10 diez años (2010-2020) en bases de datos especializadas como Scopus, Science Direct y Google Scholar, empleando ecuaciones de búsqueda con palabras claves como: "Underground water OR groundwater AND quality", "Underground water OR groundwater AND Pollution", "Use of Underground water OR Use of Groundwater", "Underground water OR groundwater AND climate change", "Underground water OR groundwater AND optimization". La información recopilada permitió definir brechas de investigación sobre temáticas asociadas al estudio del agua subterránea.

Para el manejo de la información obtenida de los motores de búsqueda de las bases de datos, se consideraron como parámetros de selección: i) las palabras claves "Underground water" y "Groundwater" debían aparecer en el título y el resumen de los artículos consultados y ii) los artículos científicos debían ser indexados. De los documentos preseleccionados, se leyó cada resumen una vez más para seleccionar los documentos que abordaban una temática asociada al agua subterránea. En el caso de que no se cumpliera con alguno de los parámetros de selección el artículo fue descartado. La Figura 1 presenta el proceso de selección de los artículos a partir de las ecuaciones de búsqueda. Los documentos seleccionados fueron leídos por completo para identificar otros estudios que incluían ejes temáticos sobre agua subterránea.

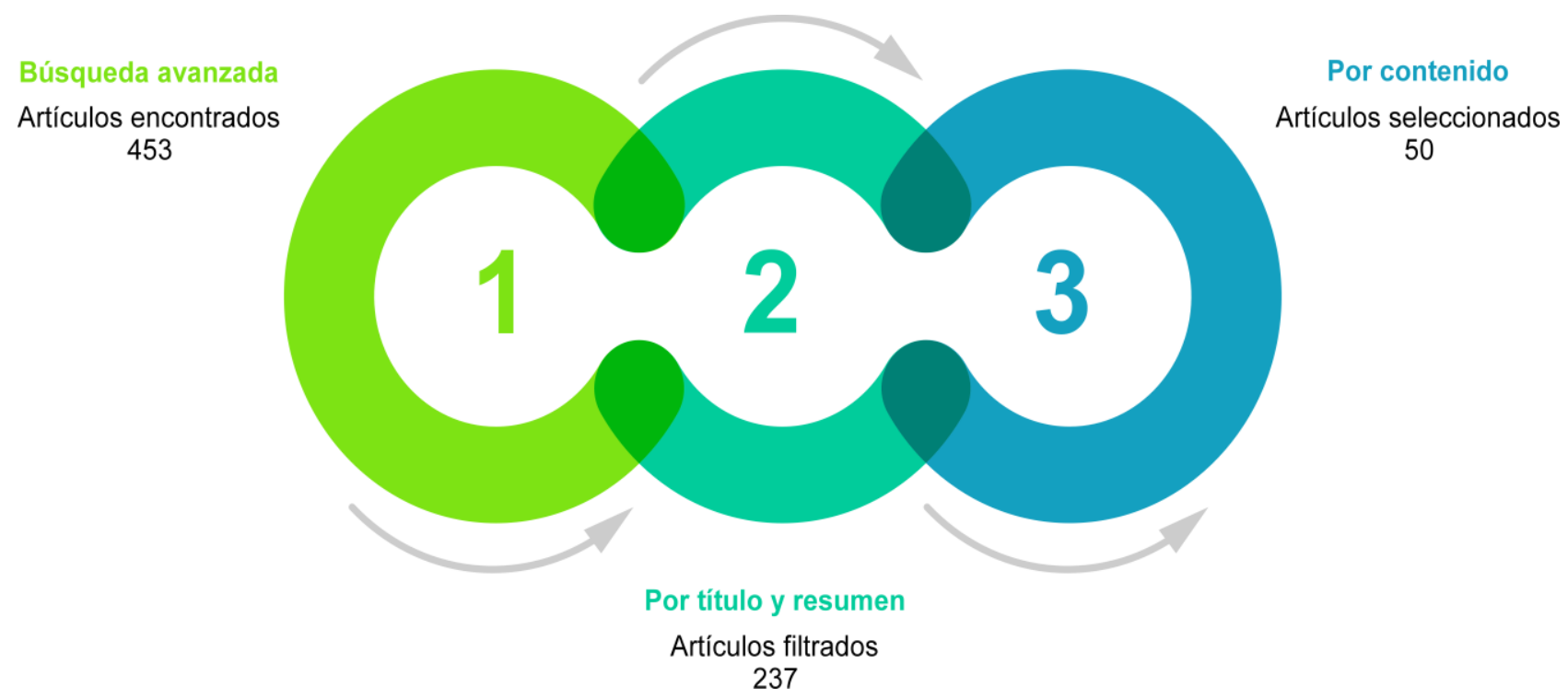

Fig. 1: Esquema del proceso de selección de los 50 artículos utilizados en el estudio 
El software Excel® se utilizó para tabular los datos de los documentos seleccionados. Los datos tabulados incluyeron: autores, año de publicación, país donde se realizó la investigación y palabras claves. De los 237 artículos, la información proporcionada por estos fue procesada mediante los softwares RefViz® y Tlab® versión libre; RefViz® se empleó para generar tablas de síntesis con los temas abordados en la literatura, lo cual permitió generar grupos con artículos que presentaban semejanza o proximidad entre sus palabras claves y permitían su compilación. Los grupos se conformaron tomando como criterio la relación de aspectos convergentes o semejanzas en los temas de los artículos mediante el análisis de la recurrencia de las palabras claves a través de la herramienta coseno de $\operatorname{RefViz}{ }^{\circledR}$. De otro lado, Tlab® se utilizó para generar mapas de co-ocurrencia de palabras en los grupos con mayor número de publicaciones para determinar las tendencias de investigación. Los mapas tienen en su eje central la palabra clave con mayor recurrencia y alrededor de esta, palabras circundantes que guardan una relación, la cual es determinada por la distancia euclidiana entre estas. La proximidad entre una palabra circundante y la central implica que una línea de investigación es más estudiada.

De los 237 artículos se seleccionaron 50 artículos de interés tomando como criterio de selección una frecuencia superior a 10 citaciones; sin embargo, también fueron incluidos aquellos artículos que no cumplieron con este criterio debido a ser de reciente publicación pero que si realizaban una contribución aplicable al contexto de estudio. Finalmente, se realizó una reflexión crítica sobre las tendencias de investigación que permita identificar brechas o vacíos de investigación en este tema.

\section{RESULTADOS Y DISCUSIÓN}

El agua subterránea se ha convertido en una importante fuente de abastecimiento de agua, entre otras razones debido al incremento en la demanda hídrica que las grandes urbes exigen para suplir sus necesidades (Subba Rao y Chaudhary, 2019). A nivel mundial un tercio de las extracciones de agua dulce corresponde a una fuente subterránea, destinada para uso agrícola (42\%), doméstico (36\%) e industrial (22\%) (Döll et al., 2012). Una de las principales preocupaciones respecto a este recurso está asociado a procesos de contaminación relacionados a actividades agrícolas, pecuarias, conexiones erradas y contaminación de fuentes superficiales que contribuyen a la recarga de acuíferos (Egbueri, 2019). A pesar de la amplia investigación e implementación de sistemas que usan agua subterránea como fuente de abastecimiento tanto en países desarrollados como en desarrollo aún se realizan esfuerzos para profundizar en aspectos como la variabilidad hidrogeoquímica del agua, índices de calidad, riesgo por contaminación y efectos por el cambio climático. Esto ha llevado a un creciente enfoque de investigación sobre la calidad del agua subterránea en todo el mundo.

\section{Literatura disponible}

Durante el periodo comprendido entre 2010 y 2020, se encontraron 237 artículos que muestran la pertinencia de ahondar en diferentes campos de investigación. La Figura 2, muestra las galaxias conformadas del procesamiento de la información con el software RefViz®.

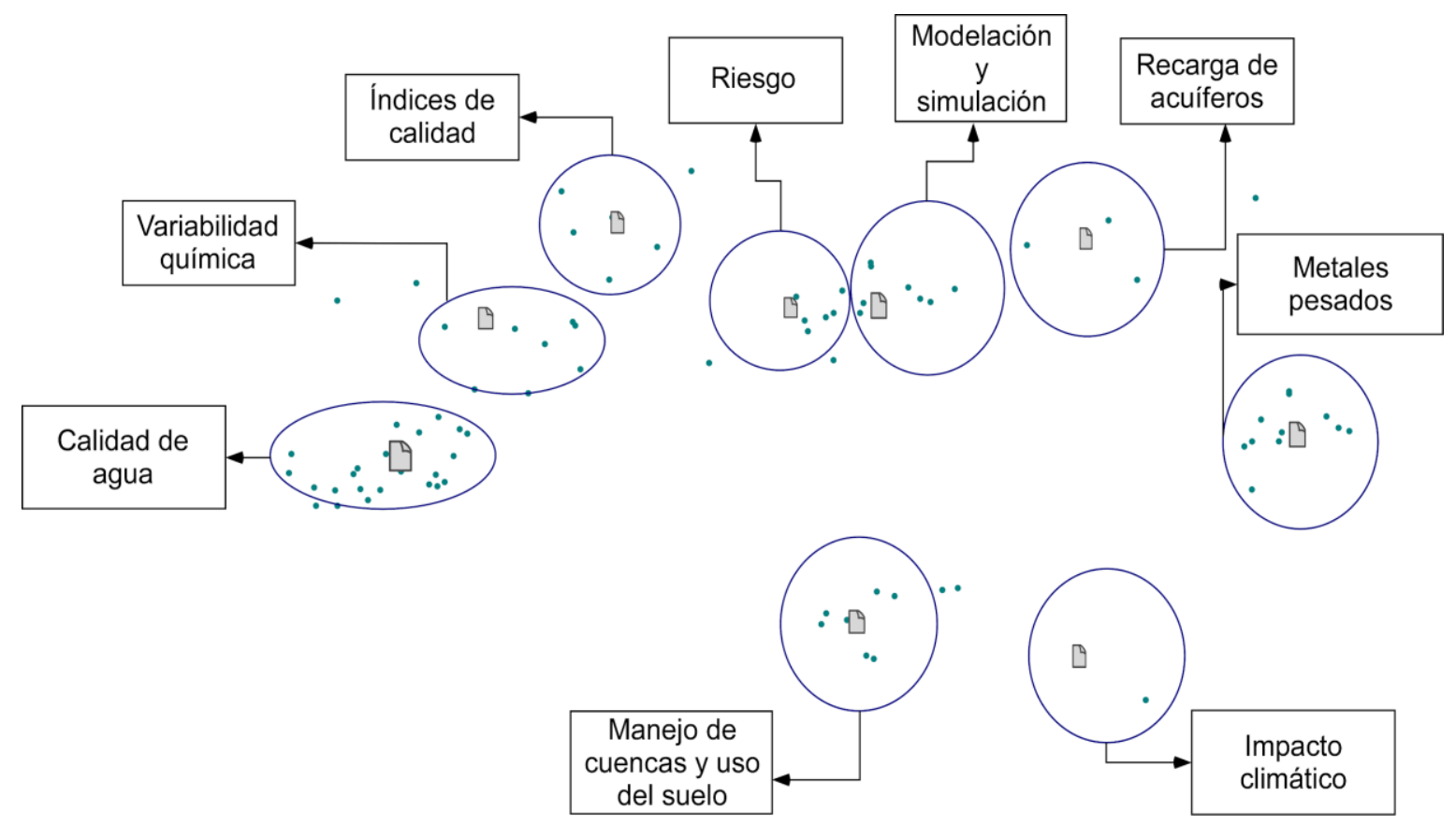

Fig. 2: Tendencias investigativas en agua subterránea en el periodo 2010-2020 
Se encontraron 9 galaxias que contienen información sobre las tendencias de investigación en la gestión del agua subterránea, los cuales fueron agrupados en 4 ejes temáticos: I) Cuencas y uso del suelo; ii) Modelado e impacto climático; iii) Estudios hidrogeológicos y iv) Calidad del agua subterránea; donde a mayor proximidad entre los puntos de cada grupo significa mayor congruencia entre los temas abordados por cada grupo. La Tabla 1 muestra cuatro grupos conformados a partir de las semejanzas y consistencias temáticas que presenta cada una de las 9 galaxias, así como el número de artículos que conforman cada eje temático y galaxia respectiva.

Tabla 1: Tendencias de investigación por eje temático

\begin{tabular}{|l|l|c|}
\hline \multicolumn{1}{|c|}{ Grupo } & \multicolumn{1}{c|}{ Galaxias } & $\begin{array}{c}\text { Número de } \\
\text { publicaciones }\end{array}$ \\
\hline 1. Cuencas y uso del suelo & Manejo de cuencas y uso del suelo & 39 \\
\hline \multirow{2}{*}{ 2. Modelado e impacto climático } & Modelación y simulación & 36 \\
\cline { 2 - 3 } & Impacto climático & 19 \\
\hline \multirow{3}{*}{ 3. Estudios hidrogeológicos } & Metales pesados & 28 \\
\cline { 2 - 3 } & Recarga de acuíferos & 18 \\
\cline { 2 - 3 } & Variabilidad química & 16 \\
\hline \multirow{3}{*}{ 4. Calidad del agua subterránea. } & Calidad de agua & 19 \\
\cline { 2 - 3 } & Índices & 13 \\
\cline { 2 - 3 } & Riesgo & 13 \\
\hline
\end{tabular}

En el grupo uno, por ejemplo, se encuentran temas como el adecuado manejo de las cuencas hidrográficas y uso del suelo. Döll et al. (2012) indican que el recurso hídrico es un sistema complejo en el cual el manejo de las cuencas tiene un efecto directo sobre la dinámica de recarga de un acuífero y por tanto, es fundamental orientar acciones que permitan maximizar la protección de las cuencas a fin de preservar el estado de los acuíferos. En este sentido, se evidencia la interdependencia de estos subsistemas y la complejidad que existe en la gestión de estos recursos. El grupo dos, muestra los estudios que adoptaron enfoques de modelación y simulación, los cuales emplean expresiones matemáticas como una herramienta para mejorar la comprensión de los sistemas de agua subterránea. Entre estos se resaltan propuestas de modelos para simular procesos agrohidrológicos regionales en cuencas con agua subterránea poco profundas; evaluación del riesgo de contaminación del agua bajó con un enfoque de alerta temprana; simulación y predicción del nivel del agua y modelos de mapeo de riesgo y vulnerabilidad (Kumar et al., 2015; Huan et al., 2020).

Recientemente, debido a los impactos ambientales generados por el cambio climático y al reconocimiento de estos como una amenaza sobre el recurso hídrico y ecosistemas, se han realizado simulaciones empleando redes neuronales artificiales como una alternativa para predecir el transporte de contaminantes como lo evidencia García et al. (2010) y así proponer estrategias de mitigación (Huan et al., 2020). Otros enfoques de modelación que han considerado el impacto del cambio climático es la recarga de acuíferos y el cálculo de las futuras demandas hídricas como fuente de abastecimiento para diferentes usos. Esto tiene relevancia desde la perspectiva de esquemas de gestión del recurso hídrico y se hace hincapié en la necesidad de desarrollar modelos de decisión con enfoque de múltiples criterios para su gestión eficaz.

El tercer grupo relaciona grandes tópicos de investigación, sin embargo, dos de ellos (recarga de acuíferos y metales pesados) son condicionantes de la variabilidad química de sustancias generalmente inorgánicas. Sulca et al. (2011), indican que los cambios dependen en un 95\% del agua meteórica y su posterior infiltración que recarga los acuíferos entendiendo que las sustancias orgánicas disueltas, aunque son frecuentes, aparecen en concentraciones mucho menores que los constituyentes inorgánicos. Igualmente, mencionan que, dentro de la heterogeneidad química, un miembro relevante pero poco estudiado son los metales pesados que son potencialmente tóxicos (e.g. $\mathrm{Cd}, \mathrm{Hg}, \mathrm{Sn}, \mathrm{Pb}, \mathrm{Zn}, \mathrm{Tl}, \mathrm{As}, \mathrm{Cu}{ }^{++}, \mathrm{Cr}, \mathrm{Ni}$ ), siendo estos componentes naturales de la corteza terrestre que no pueden ser degradados y que, según la búsqueda de información realizada en este trabajo, pueden estar presentes en el agua subterránea por tres principales mecanismos.

El primer mecanismo mencionado por Wongsasuluk et al. (2013) es aquel derivado de mineralizaciones circundantes al acuífero, siendo ésta la fuente más expresiva de contaminación geogénica; segundo, es que estos metales pesados pueden acumularse por encima de los niveles naturales en suelos agrícolas ya que la aplicación continua de agroquímicos y su posterior migración y disponibilidad de ser lixiviados recargan indeseablemente los acuíferos. Finalmente, el tercer mecanismo es provocado por agua meteórica ácida (previamente contaminada) que tiende a corroer metales como lo explica Sánchez et al. (2016), pues menciona que muchas partes y equipos de plomería como bombas, contenedores y tuberías son construidas con estos metales y podrían causar que en este proceso se incorporen iones potencialmente contaminantes 
a los acuíferos, siendo más crítico si se considera que el flujo subterráneo sirve como medio de transporte y puede provocar que estos iones lleguen a contaminar pozos, manantiales e incluso aguas superficiales recargadas por ellas.

Por último, el cuarto grupo se establece a partir de conceptos como calidad de agua, índices y riesgos; los cuales representan los esfuerzos dirigidos al cuidado y preservación de este recurso, ya que este grupo se caracteriza por tener el mayor número (\#) de publicaciones asociadas. La gestión integral de los recursos hídricos incluye la evaluación de la calidad del agua mediante la caracterización de diferentes parámetros fisicoquímicos y biológicos que permiten realizar un diagnóstico del estado del agua y los riesgos asociados de acuerdo a su uso, complementario a esto los Índices de Calidad del Agua (ICAs) son una herramienta que facilita la comunicación y entendimiento de los resultados técnicos entre los diferentes actores responsables de la gestión del agua (Torres et al., 2009).

Cuando se habla de riesgo, por su parte, es una forma sistemática de evaluar los riesgos asociados a la presencia de peligros en una fuente hídrica que facilitan la adopción de decisiones en materia de gestión de riesgos y su comunicación. Se estima que al menos 2000 millones de personas se abastecen de una fuente de agua contaminada, lo que aumenta el riesgo de contraer enfermedades infecciosas y parasitarias; además para el año 2025 la mitad de la población mundial vivirá en zonas con escasez de agua (WHO, 2019).

\section{Tendencias de investigación sobre agua subterránea}

Se identificó la calidad del agua como un área de investigación que es tendencia a nivel mundial en el lapso de los últimos diez años, esto reflejado en la cantidad de países de los diferentes continentes en donde se han realizado los estudios, en el número de publicaciones alrededor de este tema y la cantidad de citaciones de estos artículos. Así, se puede evidenciar que asegurar la calidad del agua destinada para consumo, y disminuir los riesgos asociados a sus usos se ha convertido en una preocupación en el marco de la gestión integrada del recurso hídrico a nivel mundial (Ver Figura 1). Por tanto, el monitoreo ambiental para la evaluación de la calidad de las aguas superficiales y subterráneas es sin duda uno de los principales ejes que deben considerarse en la conservación del recurso y las políticas dirigidas a su protección.

La necesidad humana de conocer su entorno y la importancia de la calidad del recurso hídrico especialmente para consumo humano, ha promovido avances significativos en el estudio de la hidrogeoquímica, permitiendo la identificación y clasificación de los constituyentes naturales del recurso. Tanto constituyentes mayoritarios como aniones $\left(\mathrm{Cl}^{-}, \mathrm{SO}^{=}\right)$y cationes $\left(\mathrm{Ca}^{++}, \mathrm{Mg}^{++}\right.$y Na$\left.{ }^{+}\right)$; algunos menores $\left(\mathrm{Mn}^{++}, \mathrm{Fe}^{++}, \mathrm{Br}^{-}\right)$y traza $\left(\mathrm{Al}^{+++}, \mathrm{Ti}^{+4}\right.$, $\mathrm{Co}^{++}, \mathrm{Cu}^{++}, \mathrm{Pb}^{++}, \mathrm{Ni}^{++}$) que resultan de interés para definir la calidad del agua como lo indica Sánchez et al. (2015).

Existe una tendencia en investigaciones respecto a la composición química del agua subterránea que según Güler et al. (2002), se encuentra dada por factores como las precipitaciones, el clima, la topografía y esencialmente la interacción agua-roca; factores que combinados crean diversos tipos de agua que cambian espacial y temporalmente. Como es descrito por Kumar et al. (2009), las concentraciones y la evolución de los constituyentes químicos de las aguas subterráneas dependen de la disponibilidad de los elementos en la roca en que se almacena o del suelo a través del cual circula el agua, las propiedades intrínsecas (e.g. presión-temperatura) en los poros del acuífero y el tiempo de reacción; sin embargo, otros autores como (Pernía et al., 1993) mencionan la relevancia de los fenómenos de contaminación antrópica.

Cuando se pretende abordar la calidad del recurso hídrico subterráneo, los investigadores han decidido en primera instancia evaluar la influencia de los eventos de recarga por infiltración bien sea de cuerpos superficiales o precipitaciones como lo desarrolla Andreu et al. (2006) en su investigación, ya que modifica la hidrogeoquímica de los acuíferos pues el agua infiltrada implícitamente contiene pequeñas cantidades de sustancias disueltas del orden de 0,2-0,4 mg/L en cada ión. En la trayectoria a través del suelo, generalmente se convierten en aguas agresivas con los silicatos y carbonatos formadores de acuíferos como lo indica Pastor y Rebollo (2010), ya que adoptan una naturaleza ácida debido a la presencia de altas concentraciones de $\mathrm{CO}_{2}$ en los poros del suelo.

Investigaciones como la de Sulca et al. (2011), indican que la composición del agua subterránea en un punto y momento determinado, dependerá esencialmente de su historia previa (atmósfera, suelo, zona saturada y no saturada) y de la relación con otras fuentes de agua. Además, se reconocen otros dos fenómenos modificadores de la composición inicial del agua subterránea durante el proceso de infiltración y tránsito por el medio hidrogeológico como sugiere Bibiano y Llanos (2015). El primer fenómeno se refiere a la ocurrencia de reacciones en las que predominan las condiciones reductoras anóxicas en la parte más externa de la zona de saturación y la presencia de algunas bacterias sulfato reductoras (e.g. Desulfovibrio desulfuricans); el 
segundo fenómeno se ejemplifica más expresivamente con la disolución y precipitación de fases minerales como calizas y dolomitas o a través de hidrólisis de silicatos.

A partir del conocimiento hidrogeoquímico descrito anteriormente se hace posible valorar el recurso hídrico subterráneo mediante el monitoreo de una serie de parámetros físicos, químicos y/o biológicos que permiten diagnosticar el estado del agua e identificar los riesgos asociados de acuerdo a su uso. Sin embargo, la interpretación de una caracterización fisicoquímica del agua puede resultar una tarea de alto grado de especialidad técnica y de difícil comprensión por fuera del ámbito técnico-científico (Mukate et al., 2019). Ante esta dificultad, el Índice de Calidad del Agua (ICA) surge como una herramienta para simplificar la interpretación de los resultados, resumiendo una gran cantidad de datos a una única expresión numérica mediante herramientas matemáticas que permiten categorizar el estado del agua de acuerdo a su calidad (Torres et al., 2009).

A mediados del siglo XX la calidad del agua fue categorizada matemáticamente integrando de un modo simple parámetros físicos, químicos y biológicos, sin embargo, tan sólo en 1970 fue construido el primer índice general de calidad del agua por Brown et al. (1970). Posteriormente, el índice Sapróbico, definido como el grado para especificar la carga de materia orgánica fácilmente degradable en corrientes de agua, fue de los primeros índices adoptados por diversos países europeos usando la base de que cada grupo de organismos tiene diferente tasa sapróbica, aunque después se determinó que este índice terminaba siendo deficiente y poco práctico (Sladecek, 1973).

A partir de los 80's varios investigadores desarrollaron índices de calidad abordando el recurso hídrico desde diferentes perspectivas y usos potenciales, sobresaliendo entre ellos Steinhart et al. (1982) que desarrollaron un nuevo índice de calidad ambiental para resumir la información técnica sobre el estado y las tendencias en los ecosistemas de los Grandes Lagos en norteamérica. Bhargava (1983) fue pionero por su trabajo desarrollado en la India, allí consideran los parámetros que amplifican la carga contaminante y cuantifican la calidad del agua en valores de 0 a 100 , refiriéndose a cero el agua extremadamente contaminada y cien el agua en óptimas condiciones.

En la década de los 90's fueron introducidos nuevos ICAs por el consejo canadiense de ministros del ambiente usado como base para informar al público e identificar cuencas hidrográficas para acciones prioritarias. Ongley y Booty (1999) en sus trabajos de finales del siglo XX se interesó por los países en desarrollo y sugirió un ICA donde relaciona tan sólo unos pocos parámetros que considera críticos, así permite fortalecer estrategias viables de control de la contaminación. Por otra parte, desde mediados de los 90's la insuficiente disponibilidad del agua superficial hizo importante conocer las características del agua subterránea, así pues, el monitoreo regular de la calidad del agua se volvió necesario para definir su uso potencial (Bharti y Katyal, 2011).

En resumen muchos ICAs han sido desarrollados y empleados para evaluar la calidad del agua tanto superficial como subterránea, ejemplo de ello podemos citar índices como ICA-NSF, ICA-Dinius, CCME-WQI, DWQI, ISQA, IWQI, GWQI, entre otros, los cuales difieren en aspectos como la cantidad y naturaleza de parámetros fisicoquímicos, el mecanismo para transformar los valores de la caracterización a una escala en común, es decir, la asignación de los subíndices (Subi), los pesos porcentuales y la estructura matemática de agregación de los Subi.

La Figura 3 presenta el diagrama de co-ocurrencia de los principales índices empleados para evaluar la calidad del agua subterránea, presentando que índices como "WQP", "GWQI"y "IWQl" (por sus siglas en inglés Water Quality Index, Ground-Water Quality Index y Integrated Water Quality Index, respectivamente) han sido ampliamente empleados a nivel mundial en diferentes estudios de calidad del agua. Aunque existe similitud entre los índices desarrollados también hay diferencias significativas en los criterios y en la metodología establecida para cada uno, lo que condiciona su uso universal dado que algunas diferencias son en función de características ambientales propias del acuífero u objetivos del estudio.

La selección de los parámetros físicos y químicos integrados al índice representa la diferencia más evidente, los cuales son definidos teniendo en cuenta diferentes aspectos relacionados al tipo de fuente y sus condiciones naturales (topográficas, climáticas, geológicas, biológicas) y antropogénicas (uso del suelo, contaminación local); o por aspectos técnicos para simplificar y agilizar el monitoreo de la calidad del agua, relacionado principalmente con la cantidad de parámetros seleccionados y la capacidad técnica de monitoreo. Por otra parte, el grado de contaminación o Subi que representa cada parámetro por sí mismo, se estima transformado el valor medido en el agua en un factor respecto a límites permisibles de la normatividad o valores de referencia a nivel internacional; o de otra manera, normalizando los valores de cada parámetro mediante tablas o gráficas previamente construidas. Finalmente, la ponderación de los parámetros para el cálculo final de un índice se puede realizar a través de diferentes funciones matemáticas de producto ponderado o suma ponderada. 


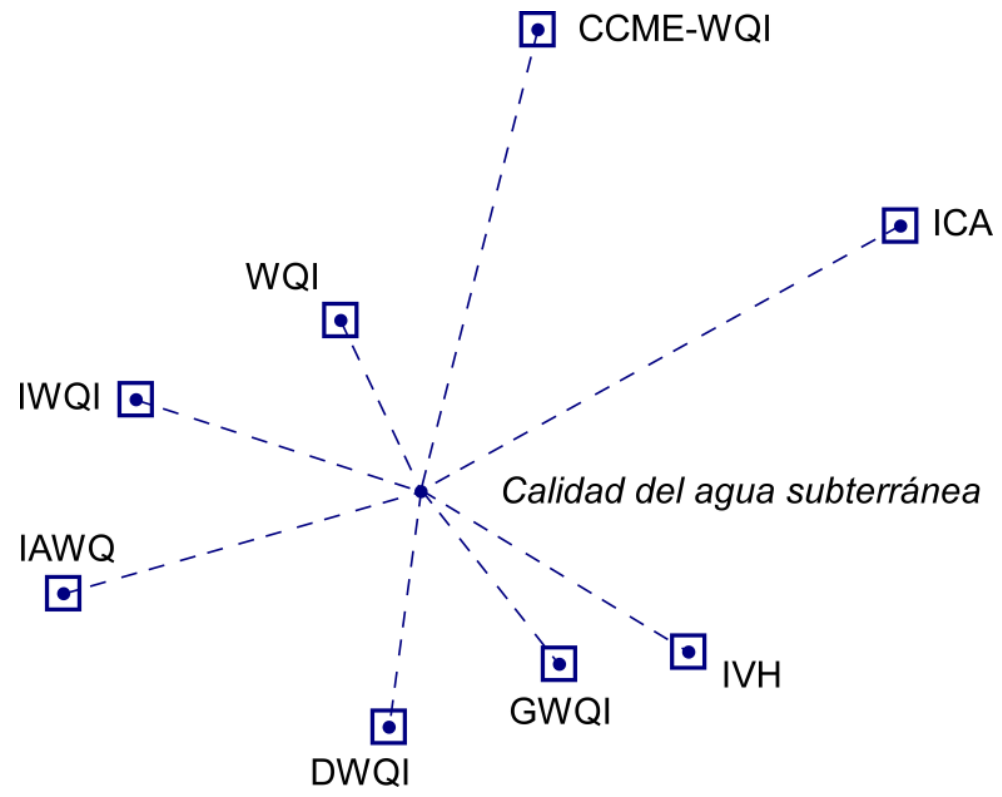

Fig. 3: Co-ocurrencias de palabras en torno a los índices de calidad

En la Tabla 2 se hace una revisión y análisis de los principales índices empleado para evaluar la calidad del agua subterránea a nivel nacional e internacional: Estos índices expuestos en la Tabla 2 emplean ecuaciones de tipo aditivo o suma ponderada que es menos sensible a variaciones extremas de las características físicas, químicas y microbiológicas del agua, entendiendo que el agua subterránea presenta variaciones menos extremas en comparación con el agua superficial.

Tabla 2: Parámetros fisicoquímicos empleados por diferentes índices

\begin{tabular}{|c|c|c|c|}
\hline & \multicolumn{3}{|c|}{ Índice } \\
\hline & WQI & GWQI & IWQI \\
\hline Parámetros & $W Q I=\sum_{i=1}^{n} S I_{i}$ & $G W Q I=\sum_{i=0}^{n} S I_{i}$ & $I W Q l i=\sum_{i=1}^{n} S I i j$ \\
\hline $\mathrm{NO}_{3}$ & $x$ & & $x$ \\
\hline $\mathrm{PO}_{4}{ }^{3-}$ & $x$ & & \\
\hline $\mathrm{Cl}^{-}$ & $x$ & $x$ & $x$ \\
\hline TDS & $x$ & $x$ & $x$ \\
\hline BOD & $x$ & & \\
\hline Cd & $x$ & & $x$ \\
\hline $\mathrm{Cr}$ & $x$ & & \\
\hline $\mathrm{Ni}$ & $x$ & & \\
\hline $\mathrm{Pb}$ & $x$ & & \\
\hline K & & $x$ & $x$ \\
\hline $\mathrm{Na}$ & & $x$ & $x$ \\
\hline $\mathrm{Ca}$ & & $x$ & $x$ \\
\hline $\mathrm{Mg}$ & & $x$ & $x$ \\
\hline $\mathrm{SO}_{4}$ & & $x$ & $x$ \\
\hline $\mathrm{pH}$ & & $x$ & $x$ \\
\hline
\end{tabular}

El IWQ es empleado por primera vez por Soltan (1999), está compuesto por nueve (9) parámetros de calidad incluyendo el monitoreo de metales pesados, seleccionados por su importancia como indicadores de evaluación de la calidad del agua con respecto a todos los usos. El cálculo del índice inicia con el cálculo de 
la calificación de calidad para cada parámetro (qi). Valores del WQI próximos a cero (0) indican ausencia o baja concentración de sustancias contaminantes, mientras que muestras de agua con graves problemas de contaminación presentan WQI por encima de cien (100).

Posteriormente, es presentado el índice GWQI por Saeedi et al. (2010) como un método simple, económico y preciso para la evaluación de la calidad del agua alrededor del contenido de iones para agua potable, basado en el análisis multivariado; se ha empleado ampliamente para evaluar la calidad del agua subterránea para consumo humano en 213 regiones del mundo. EI GWQI es un índice basado en NSF-WQI que utiliza las concentraciones estandarizadas de cada parámetro en lugar de los valores del subíndice. Inicia con la selección de ocho parámetros de cationes y aniones, se calcula la proporción de las concentraciones medidas de estos parámetros en relación a los límites de concentración permisible en la normatividad y estas fracciones calculadas son normalizadas para cada parámetro. Finalmente, con todos los parámetros normalizados, se define la importancia relativa o los pesos ponderados de los parámetros en el índice final de calidad del agua subterránea (Adimalla y Taloor, 2020).

Entre los índices más recientes se encuentra el expuesto por Mukate et al. (2019), quienes desarrollaron el índice integrado de calidad del agua (IWQI) teniendo en cuenta que la concentración de cualquier parámetro por debajo del límite deseable y por encima del límite permitido contribuirá al aumento general del valor del índice. En el WQI tradicional, los límites deseables o permisibles se evalúan como el límite permitido estándar, pero en el IWQI se consideró ambos límites, evitando la confusión en torno a la adopción de valores estándar. Basado en este concepto, IWQI se ha clasificado en 5 categorías, a saber; excelente $(<1)$, bueno $(1-2)$, marginal (2-3), pobre (3-5) e inadecuado (> 5); depende de la concentración de cationes (Ca, Mg, Na y K), aniones $\left(\mathrm{Cl}, \mathrm{SO}_{4}\right.$ y $\left.\mathrm{NO}_{3}\right)$ y otros parámetros ( $\mathrm{pH}$, TDS) presentes en las muestras de agua subterránea. La aplicación de los ICAs resulta ser un método simple y útil para llevar a cabo esta tarea, sin embargo, la calidad de los resultados obtenidos estará ligado a la adecuada selección de la metodología para calcular el índice. Además, su aplicación junto con herramientas Sistemas de información geográfica (SIG) permite realizar un monitoreo espacio-temporal de la calidad del agua y poder realizar una gestión más pertinente del recurso (Mukate et al., 2019).

Muchos de estos índices han sido desarrollados conforme las características ambientales de un acuífero en particular, sin embargo, algunos han sido adaptados y aplicados en diferentes países obteniendo resultados satisfactorios, a pesar de esto no son de aplicación universal debido a las distintas condiciones ambientales que presentan las fuentes hídricas geográficamente distanciadas, así su implementación presenta una serie de potencialidades y limitaciones que dependen en gran medida de la adecuada selección o adaptación del índice a las condiciones propias del acuífero a evaluar (Pérez, Nardini y Galindo, 2018). Por tanto, es importante conocer las bases metodológicas de cada ICA antes de aplicarlo en un sistema acuático e identificar las características y necesidades de la zona de estudio, mediante paneles de discusión entre todos los actores interesados en la gestión del recurso hídrico. La elaboración o adaptación de un indicador será la mejor opción para el uso del mismo.

\section{CONCLUSIONES}

La investigación técnico científica en torno a la gestión del agua subterránea ha tomado relevancia dado su actual y futura importancia como fuente de abastecimiento. Los resultados del presente estudio permitieron identificar las principales tendencias en los campos de investigación y sus avances. Del análisis y discusión de esta información se pueden extraer las siguientes conclusiones: 1) La gestión de cuentas y uso del suelo, la modelación e impacto climático, estudios hidrogeológicos y la calidad del agua forman los principales aspectos técnicos sobre los que se enfocan los avances de investigación, los cuales se relacionan directa 0 indirectamente en sistemas tan complejos como un acuífero. 2) La calidad del agua subterránea representa un aspecto de relevancia mundial y por lo tanto se ha convertido en uno de los campos de investigación más atendidos y con mayores avances, dentro de este campo vale la pena resaltar la implementación combinada de los ICAs junto con SIG, herramientas que aumentan la capacidad de monitorear la calidad del agua subterránea y comprender su variabilidad espacio-temporal.

\section{REFERENCIAS}

Adimalla, N., y Taloor, A. K., Hydrogeochemical Investigation of Groundwater Quality in the Hard Rock Terrain of South India Using Geographic Information System (GIS) and Groundwater Quality Index (GWQI) Techniques, https://doi.org/10.1016/j.gsd.2019.100288, Groundwater for Sustainable Development, 10, 1-16 (2020)

Andreu, J. M., García, E., y otros cinco autores, Influencia de los Eventos de Recarga en la Hidrogeoquímica del Acuífero del Ventós-Castellar (Alicante), ISSN: 0213-683X, Geogaceta, 39, 91-94 (2006).

Bibiano, L., Gárfias, J., y Llanos, H., Hidrogeoquímica y Procesos Naturales de Ablandamiento del Agua Subterránea en Sistemas Kársticos, ISSN: 2007-2422, Tecnología y Ciencias del Agua, 6(3), 57-78 (2015). 
Bhargava, D. S., Most rapid BOD Assimilation in Ganga and Yamuna Rivers, https://doi.org/10.1061/(ASCE)07339372(1983)109:1(174), J. Environ. Eng., Am. Soc. Civ. Eng., 109(1), 174-188 (1983).

Bharti, N., y Katyal, D., Water Quality Indices Used for Surface Water Vulnerability Assessment, ISSN 0976 - 4402 , Int. J. Environ. Sci., 2(1), 154-173 (2011).

Brown, R. M., McClelland, N. I., y otros dos autores, A Water Quality Index - Do We Dare, https://doi.org/10.1007/978-14684-2856-8_15, Water and Sewage Works, 117, 339- 343 (1970)

Döll, P., Hoffmann, H., y otros seis autores, Impact of Water Withdrawals from Groundwater and Surface Water on Continental Water Storage Variations, http://doi.org/10.1016/j.jog.2011.05.001, Journal of Geodynamics, 59, 143-156 (2012).

Egbueri, J. C., Evaluation and Characterization of the Groundwater Quality and Hydrogeochemistry of Ogbaru Farming District in Southeastern Nigeria, http://doi.org/10.1007/s42452-019-0853-1, SN Applied Sciences, 1(8), $851-866$ (2019).

García, I., Rodríguez J.G., y otros dos autores, Transporte de Contaminantes en Aguas Subterráneas mediante Redes Neuronales Artificiales, http://doi.org/10.1612/inf.tecnol.4349it.09, Información Tecnológica, 21(5), 79-86 (2010).

Gaviria, J.F., Soto, J., y otros dos autores, Tendencias de Investigación en la Cadena de Suministro de Residuos Sólidos Municipales, http://doi.org/10.4067/S0718-07642019000400147, Información Tecnológica, 30(4), 147-154 (2019).

Güler, C., Thyne, G. D., y otros dos autores, Evaluation of Graphical and Multivariate Statistical Methods for Classification of Water Chemistry Data, http://doi.org/10.1007/s10040-002-0196-6, Hydrogeology Journal, 10(1), 455-474 (2002).

Huan, H., Li, X., y otros cuatro autores, Groundwater Pollution Early Warning Based on QTR Model for Regional Risk Management: A Case Study in Luoyang City, China, http://doi.org/10.1016/j.envpol.2019.113900, Environmental Pollution, 1-10 (2020).

Kalhor, K., Ghasemizadeh, R., y otros dos autores, Assessment of Groundwater Quality and Remediation in Karst Aquifers: a Review, http://doi.org/10.1016/j.gsd.2018.10.004, Groundwater for Sustainable Development, 8, 104-121 (2019).

Kumar, M., Kumari, K., y otros dos autores, Hydrogeochemical Processes in the Groundwater Environment of Muktsar, Punjab: Conventional Graphical and Multivariate Statistical Approach, http://doi.org/10.1007/s00254-008-1367-0, J. Environmental Geology, 57(1), 873-884 (2009).

Kumar, P., Bansod, B. K., y otros tres autores, Index-Based Groundwater Vulnerability Mapping Models Using Hydrogeological Settings: a Critical Evaluation, http://doi.org/101007/s00254-008-1367-0, Environmental Impact Assessment Review, 51, 38-49 (2015).

Mukate, S., Wagh, V., y otros tres autores, Development of New Integrated Water Quality Index (IWQI) Model to Evaluate the Drinking Suitability of Water, http://doi.org/10.1016/j.ecolind.2019.01.034, Ecological Indicators, 101, 348-354 (2019).

Ongley, E. D., y Booty, W. G., Pollution Remediation Planning in Developing Countries: Conventional Modelling Versus Knowledge Based Prediction, http://doi.org/10.1080/02508069908692131, Water Int., 24, 1-38 (1999).

Pastor, J., y Rebollo, L. F., Características Químicas de las Aguas de Áreas de Descarga, Lixiviados Superficiales y Profundos, de Aguas de Pozos y Manantiales en las Inmediaciones de Vertederos Sellados, Estudio Multidisciplinar de Vertederos Sellados. Caracterización y Pautas de Recuperación. Editorial Universidad de Alcalá, 61-74, Alcalá, España (2010).

Pérez, J. I., Nardini, A. G., y Galindo A., Análisis Comparativo de Índices de Calidad del Agua Aplicados al Río Ranchería, La Guajira-Colombia, http://dx.doi.org/10.4067/S0718-07642018000300047, Información Tecnológica, 29 (3), 47-58 (2018).

Pernía, J. M., Cuesta, F., y otros tres autores, Las Aguas Subterráneas: Importancia y Perspectivas, Madrid, Instituto Geológico y Minero de España, 271, España (1993).

Saeedi, M., Abessi, O., y otros dos autores, Development of Groundwater Quality Index, http://doi.org/10.1007/s10661009-0837-5, Environmental Monitoring and Assessment, 163(1-4), 327-335 (2010).

Sánchez, J. A., Álvarez, T., y otros tres autores, Caracterización Hidrogeoquímica de las Aguas Subterráneas del Sur del Estado de Quintana Roo, México, ISSN: 2007-2902, Revista Mexicana de Ciencias Geológicas, 32(1), 62-76 (2015).

Sánchez, J. A., Álvarez, T., y otros tres autores, Calidad del Agua Subterránea: Acuífero Sur de Quintana Roo, México, ISSN: 2007-2422, Tecnología y Ciencias del Agua, 7(4), 76-94 (2016).

Sladecek, V., System of Water Quality from Biological Point of View. In: Mai V (ed) Archiv fur Hydrobiologie, Advances in Limnology, 7, 114-137 (1973)

Soltan, M. E., Evaluation of Ground Water Quality in Dakhla Oasis (Egyptian Western Desert), http://doi.org/10.1023/A:1005948930316, Environmental Monitoring and Assessment, 57(2), 157-168 (1999).

Steinhart, C. E., Schierow, L. J., y Sonzogni, W. C., Environmental Quality Index for the Great Lakes, http://doi.org/10.1111/j.1752-1688.1982.tb00110.x, Water Resour. Bull., 18(6), 1025-1031 (1982).

Subba Rao, N., y Chaudhary, M., Hydrogeochemical Processes Regulating the Spatial Distribution of Groundwater Contamination, Using Pollution Index of Groundwater (PIG) and Hierarchical Cluster Analysis (HCA): A Case Study, http://doi: 10.1016/j.gsd.2019.100238, Groundwater for Sustainable Development, 9, 1-14 (2019). 
Sulca, P. D., Importancia de la Hidroquímica en la Prospección y Exploración de Aguas Subterráneas, tomado de X Encuentro científico internacional de verano, ECI, Instituto Geológico Minero y Metalúrgico, Perú (2011).

Torres, P., Cruz, C. H., y Patiño, P., Índices de Calidad de Agua en Fuentes Superficiales Utilizadas en la Producción de Agua para Consumo Humano. Una Revisión Crítica, ISSN: 1692-3324, Revista Ingenierías Universidad de Medellín, 8(15), 79-94 (2009).

WHO, Drinking-Water. Available at: https://www.who.int/news-room/fact-sheets/detail/drinking-water, (2019).

Wongsasuluk, P., Chotpantarat, S., y otros dos autores, Heavy Metal Contamination and Human Health Risk Assessment in Drinking Water from Shallow Groundwater Wells in an Agricultural Area in Ubon Ratchathani Province, Thailand, http://doi.org/10.1007/s10653-013-9537-8, Environ Geochem Health, 36(1), 169-82 (2013). 